\title{
A Simple Tool to Predict End-Stage Renal Disease within 1 Year in Elderly Adults with Advanced Chronic Kidney Disease
}

\author{
Paul E. Drawz, MD, MHS, MS, * Puja Goswami, MD, ${ }^{\dagger}$ Reem Azem, BA, ${ }^{\star}$ Denise C. Babineau, \\ $\mathrm{PhD},{ }^{*}$ and Mabboob Rahman, MD, MS $S^{t s}$
}

OBJECTIVES: To develop and validate a model to predict 1-year risk of end-stage renal disease (ESRD) in elderly subjects with advanced chronic kidney disease (CKD).

DESIGN: Retrospective.

SETTING: Veterans Affairs Medical Center.

PARTICIPANTS: Individuals aged 65 and older with CKD with an estimated glomerular filtration rate (eGFR) less than $30 \mathrm{~mL} / \mathrm{min}$ per $1.73 \mathrm{~m}^{2}$.

MEASUREMENTS: The outcome was ESRD within 1 year of the index eGFR. Cox regression was used to develop a predictive model (Veterans Affairs (VA) risk score) that was validated in a separate cohort.

RESULTS: Of the 1,866 participants in the developmental cohort, 77 developed ESRD. Risk factors for ESRD in the final model were age, congestive heart failure, systolic blood pressure, eGFR, potassium, and albumin. In the validation cohort, the $\mathrm{C}$ index for the VA risk score was 0.823. The risk for developing ESRD at 1 year from lowest to highest tertile was $0.08 \%, 2.7 \%$, and $11.3 \%$ $(P<.001)$. The C-index for the recently published Tangri model in the validation cohort was 0.780 .

CONCLUSION: A new model using commonly available clinical measures shows excellent ability to predict the onset of ESRD within the next year in elderly adults. The Tangri model also had good predictive ability. Individuals and physicians can use these risk models to inform decisions regarding preparation for renal replacement therapy in individuals with advanced CKD. J Am Geriatr Soc 61:762-768, 2013.

From the "Division of Renal Diseases and Hypertension, University of Minnesota, Minneapolis, Minnesota; ${ }^{\dagger}$ Division of Nephrology, University of Michigan, Ann Arbor, Michigan; ${ }^{\ddagger}$ Case Western Reserve University, Cleveland, Ohio; and ${ }^{\S}$ Division of Nephrology and Hypertension, Louis Stokes Cleveland Veterans Affairs Medical Center, University HospitalsCase Medical Center, Cleveland, Ohio.

Address correspondence to Dr. Paul E. Drawz, Department of Medicine, Division of Renal Diseases and Hypertension, 717 Delaware Street SE, Suite 353, Minneapolis, MN 55414. E-mail: draw0003@umn.edu

DOI: $10.1111 /$ jgs. 12223
Key words: chronic renal insufficiency; aging; chronic kidney failure; hypertension

$\mathrm{C}$ hronic kidney disease (CKD) affects more than 26 million Americans. ${ }^{1}$ The prevalence of CKD has increased in recent years, particularly in the elderly population. ${ }^{1}$ Approximately half of all Americans aged 70 and older have been diagnosed with CKD. ${ }^{1}$ Individuals with CKD are at risk for metabolic complications, cardiovascular disease, end-stage renal disease (ESRD), and all-cause mortality. ${ }^{2,3}$ The risk of ESRD and all-cause mortality that accompanies CKD differs according to age; older individuals are more likely to die than progress to ESRD., ${ }^{4,5}$ The rate of decline in renal function also varies and is affected by individual characteristics. ${ }^{6}$ This variation in risk has important implications for individuals with advanced CKD in whom preparation for renal replacement therapy is an essential aspect of management.

The National Kidney Foundation Kidney Disease Outcomes Quality Initiative guidelines recommend preparation for renal replacement therapy in individuals with an estimated glomerular filtration rate (eGFR) of less than $30 \mathrm{~mL} / \mathrm{min}$ per $1.73 \mathrm{~m}^{2}{ }^{7}$ Before initiating dialysis access placement, individuals should receive education regarding ESRD treatment options, including no treatment, peritoneal dialysis, transplantation, and hemodialysis. For individuals choosing hemodialysis $(>90 \%$ in the United States), the guidelines recommend placement of a fistula 6 months before initiation of dialysis. ${ }^{7}$ Although the timing of initiation of dialysis is controversial and has changed recently, no specific tools are available to help physicians and individuals with the difficult decisions related to preparation for hemodialysis. Planning for hemodialysis access takes approximately 1 year and includes referral to vascular surgery, vein mapping, surgery, and access maturation. Therefore, a tool to predict 
1-year risk of ESRD would be clinically useful, especially in elderly adults, given their lower overall risk of ESRD. Elderly adults at low risk for ESRD could be spared unnecessary tests and surgical procedures, whereas those at high risk could be referred for access placement.

Previous studies have evaluated risk factors for ESRD in various populations, but no prediction model is available to evaluate risk for ESRD at 1 year in older adults with advanced CKD. The purpose of this study was to develop and validate a prediction model for ESRD at 1 year in elderly adults with advanced CKD.

\section{METHODS}

A retrospective study was performed at the Louis Stokes Cleveland Veterans Affairs (VA) Medical Center. The institutional review board approved the study. Inclusion criteria were aged 65 and older and an index outpatient eGFR of less than $30 \mathrm{~mL} / \mathrm{min}$ per $1.73 \mathrm{~m}^{2}$ between January 1 , 2002, and January 1, 2009. Because individuals are not likely referred for renal replacement planning on the basis of one low eGFR, an outpatient eGFR of less than $30 \mathrm{~mL} / \mathrm{min}$ per $1.73 \mathrm{~m}^{2}$ in the year before the index eGFR was required. Finally, to fulfill criteria for CKD, an outpatient eGFR of less than $60 \mathrm{~mL} / \mathrm{min}$ per $1.73 \mathrm{~m}^{2}$ between 90 and 730 days before the index GFR was also required. ${ }^{8}$ A single eGFR of less than $30 \mathrm{~mL} / \mathrm{min}$ per $1.73 \mathrm{~m}^{2}$ between 90 and 365 days before the index eGFR could fulfill the latter two criteria. The first eligible index eGFR was chosen for individuals with multiple eligible eGFR values. Individuals with a kidney transplant and those receiving renal replacement therapy were excluded. The developmental cohort included individuals seen in Cleveland. A separate validation cohort included all other individuals from the Greater Ohio Veterans Integrated Service Network (VISN) 10.

Baseline data were collected from the VISN 10 database and included demographic information, blood pressure (BP) measurements, past medical history, medication use, and laboratory values. All data were obtained from the 2 years before the index eGFR. When multiple measurements were present, the most-recent value was selected. Proteinuria was defined as an albumin to creatinine ratio greater than $300 \mathrm{mg} / \mathrm{g}$, a urinalysis with 2 or greater proteinuria, or a urinalysis with more than 30 $\mathrm{mg} / \mathrm{dL}$ of protein. Comorbidities were defined as diabetes mellitus ( $\geq 2$ International Classification of Diseases, Ninth Revision (ICD-9) codes 250-250.99 or $\geq 2$ diabetic medications filled before the index eGFR), coronary artery disease ( $\geq 2$ ICD-9 codes 410-414.99, 429.2), cancer ( $\geq 2$ ICD-9 codes 140-208, 230-234, V10), congestive heart failure (CHF; $\geq 2$ ICD-9 codes 398.91, 402.01, 402.11, $402.91,404.01,404.03,404.11,404.13,404.91,404.93$, 414.8, or between 428 and 428.99), and chronic obstructive pulmonary disease (COPD; $\geq 2$ ICD-9 codes $491-494,496,510)$. VA ICD-9 codes have been shown to have moderate sensitivity $(>80 \%)$ and excellent specificity $(>90 \%))^{9,10}$

The primary outcome was ESRD within 1 year of the index eGFR. ESRD was defined as the need for chronic renal replacement therapy as determined according to chart review blinded to the predictors in the final model.
Individuals were not considered to require chronic renal replacement therapy in instances of acute kidney injury if they recovered renal function or did not survive to hospital discharge. Chart reviews were conducted to evaluate eligibility and to ascertain the date of initiation of dialysis. The medical record was reviewed to adjudicate ESRD for all subjects with a hemodialysis note, an ICD-9 code for ESRD (585.5, 585.6, 792.5, V42, V45.1, V56, V56.1, V56.2, V56.31, V56.32, or V56.8), an eGFR of less than $15 \mathrm{~mL} / \mathrm{min}$ per $1.73 \mathrm{~m}^{2}$ (at baseline or during follow-up), or previously identified as having ESRD in the database. The date of initiation of dialysis was the date of the first chronic dialysis at the VA or the approximate date of initiation as determined from clinical notes if dialysis was started at an outside facility. Initiation of dialysis was at the discretion of the individual and the nephrologist and was not affected by this retrospective study. No subjects received a kidney transplant.

\section{Statistical Analyses}

Baseline characteristics in the developmental and validation samples are described using summary statistics. Time to ESRD was determined according to chart review as described above, with right censoring if subjects died or did not have ESRD. A final prediction model, the VA risk score, was developed using Cox proportional hazards models. Variables associated with risk of ESRD in unadjusted analyses were included in the base model, and age, sex, race, diabetes mellitus, CHF, COPD, cancer, angiotensin-converting enzyme inhibitor (ACEI) or angiotensin receptor blocker (ARB) use, proteinuria, index eGFR, slope of eGFR decline in the 24 months before index eGFR, blood urea nitrogen, serum bicarbonate, potassium, calcium, phosphorus, hemoglobin, albumin, and systolic and diastolic BP were entered based on their biological plausibility as predictors based on previous studies.

A final model was developed using backward stepwise selection based on the Akaike information criterion (AIC) and bootstrap sampling $(\mathrm{N}=100)$. Interaction terms were evaluated. Nonlinearity was evaluated using restricted cubic splines. Colinearity was assessed by evaluating variance inflation factors. The proportional hazards assumption was tested using cox.zph in R (www.Rproject.org), and no violations were detected. There were no missing data for most variables and fewer than $10 \%$ missing data except for phosphorus, which was unavailable for $37 \%$ of subjects. Primary analyses in the developmental cohort were conducted using complete cases $(82 \%$ complete when phosphorus not considered). Secondary analyses were conducted after imputing missing data using single imputation. In the validation cohort, missing data were imputed before validation using single imputation to compare the new risk score with the Tangri score, which includes phosphorus. ${ }^{11}$

A risk score based on the final model was calculated for subjects in the validation cohort, and model performance was evaluated using the Harrell C-index. Calibration was assessed by graphing the predicted and observed rates according to decile of risk score, and a risk score based on the work by Tangri and colleagues was calculated and performance evaluated by calculating Harrell 
C-index for the developmental and validation cohorts. ${ }^{11}$ The Tangri model was chosen because it was the mostrecent model developed to predict ESRD. Net reclassification improvement from Tangri's model to the VA risk score was calculated. ${ }^{12}$ Secondary analyses compared the risk model developed using Cox models to models developed using penalized estimation and competing risks regression. ${ }^{13}$ Statistical analyses were conducted using $\mathrm{R}$ 2.12.2 (www.R-project.org).

\section{RESULTS}

There were 151,783 individuals at the Cleveland VA with an outpatient eGFR between January 1, 2002, and January 1,2009 . Of these, 7,204 had at least one eGFR of less than $30 \mathrm{~mL} / \mathrm{min}$ per $1.73 \mathrm{~m}^{2} ; 1,866 \mathrm{had}$ an eligible index eGFR of less than $30 \mathrm{~mL} / \mathrm{min}$ per $1.73 \mathrm{~m}^{2}$, were aged 65 and older, and did not have pre-existing ESRD or a kidney transplant and were included in the present analyses (Figure 1).

Medical records were reviewed for 541 individuals who met at least one criterion for chart review (hemodialysis note, ICD-9 code, eGFR $<15 \mathrm{~mL} / \mathrm{min}$ per $1.73 \mathrm{~m}^{2}$ at baseline or during follow-up or previously noted to have ESRD in the database). To evaluate the sensitivity of these criteria for identifying ESRD, the medical record was reviewed for an additional 20 individuals with the lowest follow-up eGFR of greater than $15 \mathrm{~mL} / \mathrm{min}$ per $1.73 \mathrm{~m}^{2}$ and for 15 random additional subjects whose lowest follow-up eGFR was between 16 and $30 \mathrm{~mL} / \mathrm{min}$ per $1.73 \mathrm{~m}^{2}$. No cases of ESRD were identified in these additional chart reviews, an indication that the criteria for chart review were sensitive.

Of the 1,866 participants in the developmental cohort, 77 developed ESRD, as adjudicated according to investigators blinded to VA risk score, and 276 died. The rate of ESRD did not change over time. The baseline characteristics for subjects in the developmental and validation cohorts are shown in Table 1. The average decline in GFR during the 2 years before the index eGFR was $6.9 \mathrm{~mL} / \mathrm{min}$ per $1.73 \mathrm{~m}^{2} /$ year $(95 \%$ confidence interval $=-5.9-25.8)$. A number of factors were associated with greater risk of ESRD, including younger age, black race, CHF, high BP, low eGFR, proteinuria, and low bicarbonate (Table 2).

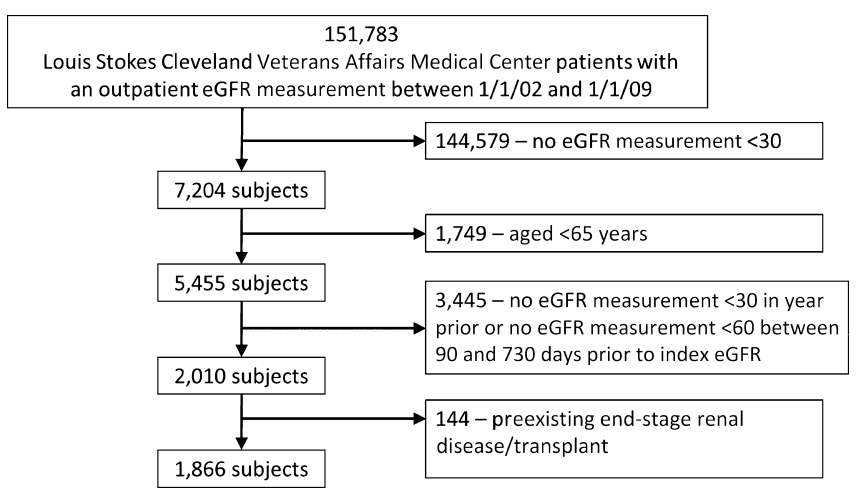

Figure 1. Participant flow for developmental cohort. eGFR $=$ estimated glomerular filtration rate.
Table 1. Baseline Characteristics of the Developmental and Validation Cohorts

\begin{tabular}{|c|c|c|}
\hline Characteristic & $\begin{array}{l}\text { Developmental } \\
\text { Cohort, } \\
n=1,866\end{array}$ & $\begin{array}{l}\text { Validation } \\
\text { Cohort, } \\
n=819\end{array}$ \\
\hline Developed ESRD & $77(4.1)$ & $33(4.0)$ \\
\hline Died without developing ESRD & $276(14.8)$ & $178(21.7)$ \\
\hline Age, mean \pm SD & $77.5 \pm 6.4$ & $78.1 \pm 6.4$ \\
\hline Black, n (\%) & $202(11.8)$ & $69(8.4)$ \\
\hline Male, n (\%) & $1,773(95)$ & $801(97.8)$ \\
\hline \multicolumn{3}{|l|}{ Past medical history, $\mathrm{n}(\%)$} \\
\hline Diabetes mellitus & $922(49.4)$ & $307(37.5)$ \\
\hline Coronary artery disease & $242(13.0)$ & $99(12.1)$ \\
\hline Congestive heart failure & $188(10.1)$ & $88(10.7)$ \\
\hline Cancer & $412(22.1)$ & $221(27.0)$ \\
\hline $\begin{array}{l}\text { Average of last } 5 \text { clinic systolic } \\
\text { blood pressure measurements, } \\
\mathrm{mmHg} \text {, mean } \pm S D\end{array}$ & $144.1 \pm 19.8$ & $138.8 \pm 19.2$ \\
\hline $\begin{array}{l}\text { Index eGFR, } \mathrm{mL} / \mathrm{min} \text { per } \\
1.73 \mathrm{~m}^{2}, \text { mean } \pm \mathrm{SD}\end{array}$ & $25.0 \pm 4.3$ & $24.9 \pm 4.3$ \\
\hline $25.0-30.0$ & $1,143(61.3)$ & $511(62.4)$ \\
\hline $20.0-24.9$ & $499(26.7)$ & $194(23.7)$ \\
\hline$<20$ & $224(12.0)$ & $114(13.9)$ \\
\hline Proteinuria, n (\%) & $693(37.1)$ & $316(38.6)$ \\
\hline Potassium, $\mathrm{mEq} / \mathrm{L}$, mean $\pm \mathrm{SD}$ & $4.68 \pm 0.6$ & $4.66 \pm 0.6$ \\
\hline Bicarbonate, $\mathrm{mEq} / \mathrm{L}$, mean $\pm \mathrm{SD}$ & $24.2 \pm 3.9$ & $25.5 \pm 4.5$ \\
\hline Serum albumin, $\mathrm{g} / \mathrm{dL}$, mean \pm SD & $3.83 \pm 0.5$ & $3.76 \pm 0.5$ \\
\hline Calcium, mg/dL, mean \pm SD & $9.10 \pm 0.6$ & $9.01 \pm 0.6$ \\
\hline Phosphorus, $\mathrm{mg} / \mathrm{dL}$, mean $\pm \mathrm{SD}$ & $3.80 \pm 0.7$ & $3.81 \pm 0.9$ \\
\hline $\begin{array}{l}\text { Angiotensin-converting enzyme } \\
\text { inhibitors or angiotensin receptor } \\
\text { blocker use, } \mathrm{n}(\%)\end{array}$ & $1,215(65.1)$ & $455(55.6)$ \\
\hline
\end{tabular}

Conversion factors for units: estimated glomerular filtration rate (eGFR) in $\mathrm{mL} / \mathrm{min}$ per $1.73 \mathrm{~m}^{2}$ to $\mathrm{mL} / \mathrm{s}$ per $1.73 \mathrm{~m}^{2} \times 0.01667$; serum albumin in $\mathrm{g} / \mathrm{dL}$ to $\mathrm{g} / \mathrm{L} \times 10$; serum calcium in $\mathrm{mg} / \mathrm{dL}$ to $\mathrm{mmol} / \mathrm{L} \times 0.2495$; serum phosphorus in $\mathrm{mg} / \mathrm{dL}$ to $\mathrm{mmol} / \mathrm{L} \times 0.3229$. No conversion necessary for serum potassium or bicarbonate in $\mathrm{mEq} / \mathrm{L}$ to $\mathrm{mmol} / \mathrm{L}$.

$\mathrm{SD}=$ standard deviation; ESRD $=$ end stage renal disease.

The final model included index eGFR, age, CHF, average of last five clinic systolic BP measurements, most recent potassium and albumin, and interactions between age and eGFR and eGFR and CHF (Table 2). Similar results were obtained when $\mathrm{CHF}$ was defined according to one or more ICD-9 codes and when death was treated as a competing risk (data not shown). ${ }^{14}$ Low potassium remained a significant risk factor when ACEI, ARB, and diuretic use were forced into the model. The bootstrapcorrected C-index in the developmental cohort was 0.854 , indicating excellent predictive ability. There was no significant nonlinearity or colinearity. The formula for calculating the risk for ESRD at 1 year based on the coefficients from the final Cox model is shown in Appendix 1. Secondary analysis after imputing missing data produced a final model with the same predictors. A model developed using penalized estimation did not improve predictive ability (net reclassification improvement $=-3.2 \% ; \quad P=.34$ ). Hazard ratios were similar when calculated using Cox proportional hazards regression and competing risks regression (Table S1).

There were 819 participants in the validation cohort, of whom 33 developed ESRD and 178 died. A risk score based on the formula in Appendix 1 was calculated for 
Table 2. Risk of End-Stage Renal Disease in the Developmental Cohort

Hazard Ratio

Variable

$\left(95 \%\right.$ Confidence Interval) ${ }^{\text {b }}$

\begin{tabular}{lll}
\hline Age & $0.71(0.56-0.90)$ & $0.60(0.42-0.86)$ \\
Black & $2.72(1.59-4.65)$ & \\
Diabetes mellitus & $1.18(0.75-1.85)$ & \\
Congestive heart failure & $2.59(1.50-4.50)$ & $4.72(2.24-9.94)$ \\
Average of last five clinical & $1.33(1.07-1.66)$ & $1.41(1.12-1.77)$ \\
SBP measurements & & \\
eGFR & $0.42(0.37-0.48)$ & $0.31(0.25-0.37)$ \\
Proteinuria & $2.41(1.53-3.79)$ & \\
Urea nitrogen & $1.55(1.32-1.82)$ & \\
Potassium & $0.78(0.62-0.98)$ & $0.74(0.58-0.93)$ \\
Bicarbonate & $0.78(0.62-0.99)$ & \\
Albumin & $0.65(0.53-0.80)$ & $0.71(0.56-0.89)$ \\
Calcium & $0.67(0.57-0.78)$ & \\
Phosphorus & $1.54(1.23-1.93)$ & \\
Hemoglobin & $0.53(0.42-0.67)$ & \\
Angiotensin-converting & $0.79(0.50-1.25)$ & \\
enzyme inhibitors or & & \\
angiotensin receptor & & \\
blocker use & & \\
\hline
\end{tabular}

a Estimated glomerular filtration rate (eGFR) by congestive heart failure interaction term $P=.009$; eGFR by age interaction term $P=.01$. Adjusted to median values: eGFR of 26.1 , no heart failure, and age of 77.7 years.

${ }^{\mathrm{b}}$ Associated with a 1-standard deviation increase for continuous variables (6.4 years for age, $19.8 \mathrm{mmHg}$ for systolic blood pressure (SBP), $0.62 \mathrm{mEq} / \mathrm{L}$ for potassium, and $0.46 \mathrm{~g} / \mathrm{dL}$ for albumin).

subjects in the validation cohort. The risk for developing ESRD at 1 year was $0.08 \%$ in participants in the lowest tertile (VA risk score $<0.7 \%$ ), $2.7 \%$ in the middle tertile, and $11.3 \%$ in the highest tertile (VA risk score $>2.9 \%$; $P<.001$; Figure 2A). The risk for ESRD at 1 year for subjects in the highest quintile (VA risk score $>5.6 \%$ ) was $14 \%$. The C-index for the risk score in the validation sample was 0.823 , indicating excellent predictive ability.

A recently published risk score (Tangri Model 6) was calculated for subjects in the development and validation cohorts. ${ }^{11}$ The C-index for the Tangri model was 0.780 in both cohorts, indicating very good predictive ability. ESRD event-free survival in the validation sample for subjects according to tertile of Tangri risk score is shown in Figure $2 \mathrm{~B}$. Net reclassification improvement based on tertiles from the Tangri model to tertiles based on the VA risk score was $5.9 \%(P=.45)$.

\section{DISCUSSION}

A model to assess risk for ESRD at 1 year in elderly adults with advanced CKD was developed and validated. The model included age, CHF, systolic BP, eGFR, potassium, and albumin and had excellent predictive ability in the validation cohort (C-index 0.823). A recently published model for predicting ESRD also had very good predictive ability in the developmental and validation cohorts (C-index 0.78). ${ }^{11}$ These models allow for identification of individuals at low risk of progression to ESRD within 1 year.

In elderly adults, CKD is an important and growing public health problem. Optimal clinical management of CKD requires assessment of the risk for development of ESRD. Previous studies have shown that the risk of ESRD associated with advanced CKD is attenuated in elderly adults. ${ }^{4,5,11,14-18}$ The risk of ESRD evaluated in a population of U.S. veterans was similar to that reported here. ${ }^{4}$ At all levels of eGFR, risk of ESRD decreased with advancing age. ${ }^{4}$ This lower risk for ESRD is in part due to the greater competing risk for all-cause mortality, and elderly adults are less likely to receive renal replacement therapy even when they advance to Stage V CKD; this may be due in part to clinical uncertainty regarding the competing risks for death and ESRD in elderly adults with advanced CKD. ${ }^{19}$ Given the lower risk for ESRD, the current study sought to develop a clinically meaningful tool to assess risk of progression to ESRD in elderly adults with advanced CKD. The risk score could be used to inform decisions regarding renal replacement therapy such as referrals and procedures for dialysis access placement.

The VA risk score includes variables previously identified as risk factors for renal function decline
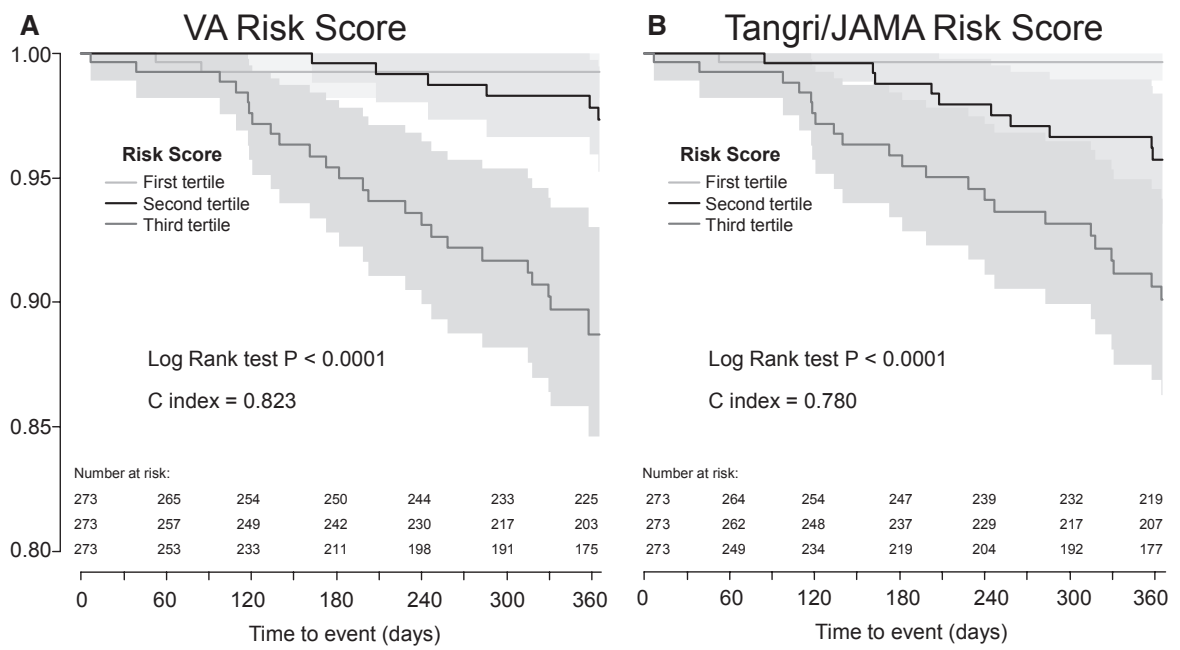

Figure 2. End-stage renal disease-free survival in the validation cohort. (A) Veterans Affairs (VA) risk score. (B) Tangri risk score. 
and ESRD: younger age, $4,5,11,15-18$ low baseline eGFR, ${ }^{11,14-17,20-22}$ high clinical systolic $\mathrm{BP},{ }^{5,14} \mathrm{CHF}^{23}$ and low serum albumin. ${ }^{11,21} \mathrm{CHF}$ may predispose to ESRD through episodes of acute kidney injury from volume changes associated with episodes of decompensated heart failure, although CHF may also be a marker of underlying cardio- and renovascular disease. Low potassium may be a marker of poor nutritional status and was associated with low albumin. Alternatively, low potassium may simply be a marker of other risk factors; in the developmental cohort, low potassium was associated with black race, low eGFR, low albumin, diuretic use, and lack of ACEI and ARB use but not CHF or systolic BP. A notable exclusion from the final model is proteinuria, a well-defined risk factor for ESRD. ${ }^{5,11,14,17,20-22}$ The lack of standard assessment may play a role- $10 \%$ of subjects had no assessment for proteinuria, and a urine albumin to creatinine ratio was available for fewer than one-third of subjects. Therefore, measurement of proteinuria in a majority of subjects was based on semiquantitative methods that are dependent on urine concentration and are not as accurate an assessment of proteinuria.

In addition to developing and validating a new model, the predictive ability of a recently published risk score was also evaluated (Tangri Model 6). ${ }^{11}$ The Tangri model and the VA risk score include eGFR, age, and serum albumin. The Tangri model also includes urine albumin to creatinine ratio and serum phosphorus, bicarbonate, and calcium. There are other important differences between the two scores. Tangri and colleagues provided a model to predict risk over a 5-year time period (compared with 1 year in this study), and their cohort included individuals with a baseline eGFR less than $60 \mathrm{~mL} / \mathrm{min}$ per $1.73 \mathrm{~m}^{2}$, whereas the current study included only those with an eGFR less than $30 \mathrm{~mL} / \mathrm{min}$ per $1.73 \mathrm{~m}^{2}$. A calculator for predicting 2-year risk of ESRD based on the Tangri model is available online. ${ }^{11}$ The Tangri model does not include BP, a variable that is included in the current model. This may be due to measurement based on only one clinic BP at the time of the initial nephrology visit in the study by Tangri. For the current study, BP was based on an average of up to five clinic measurements before the index GFR, probably a more-accurate assessment of BP. Despite the differences in cohort characteristics and length of follow-up, the Tangri model had good predictive ability in the developmental and validation cohorts.

Previous investigators have developed prediction models for a variety of kidney disease outcomes, including incident $\mathrm{CKD}^{24,25}$ progression of CKD,${ }^{26,27}$ and ESRD, ${ }^{5,11,15-17,20-22,28}$ but the models have limited utility for management of advanced CKD. Many of the models predict outcomes other than ESRD. ${ }^{24-27}$ In addition, the period over which ESRD is predicted ranges from 2.5 to 10 years, a longer time frame than required for access placement. ${ }^{5,15-17,20-22,28}$ Finally, some models include individuals with normal kidney function or mild CKD. ${ }^{11,15,16,20-22,28}$ Therefore, although previous investigators evaluated risk factors for ESRD in various populations, before the current study, no prediction model was available to evaluate risk of ESRD at 1 year in older adults with advanced CKD.
This prediction model has a number of strengths. First, the 1-year time period over which risk for ESRD was assessed is clinically meaningful in that it is approximately the time required for preparation for hemodialysis. Previous prediction models have included follow-up ranging from 2.5 to 10 years. ${ }^{5,15-17,20-22,28}$ The current model also included only subjects in whom planning for renal replacement therapy is recommended: those with advanced CKD $\left(\mathrm{eGFR}<30 \mathrm{~mL} / \mathrm{min}\right.$ per $\left.1.73 \mathrm{~m}^{2}\right){ }^{7}$ Another strength is the use of commonly available data from "real-world settings" and the development of a tool to help clinicians and individuals "choose the option that best fit(s) the individual's needs and preferences": two of the pillars of comparative effectiveness research. ${ }^{29}$ The VA risk score could be incorporated into electronic medical records, similar to the reporting of eGFR with creatinine results. The current study included a large cohort of individuals with advanced CKD. Finally, a significant strength was the validation of the prediction model in a separate cohort. ${ }^{30}$

A few limitations need to be considered. As with most VA studies, the cohort was predominantly male. The retrospective nature of the study precluded formal assessment of comorbidities and prospective collection of urine and serum samples for laboratory analyses. The diagnosis of heart failure was based on ICD-9 codes rather than echocardiographic examination. ICD-9-based diagnosis allows for automated risk score calculation using electronic medical records. Time to ESRD was adjudicated using chart review; confirmation by linkage to the U.S. Renal Data System was not performed, but the authors are fairly certain about the specificity of the diagnosis, and the chance that incident ESRD cases were "missed" is low. Proteinuria was not found to be a predictor in adjusted models despite strong evidence from prior studies demonstrating it to be a predictor of ESRD. Finally, although the prediction model was evaluated in a validation cohort, similarities between the development and validation cohorts necessitate further validation in cohorts with varying characteristics.

This study makes an important contribution and provides a new tool that can be used in the clinical setting. The excellent predictive ability of the model will provide clinicians and patients with much needed information as they contemplate the multitude of decisions surrounding renal replacement therapy, a process that is difficult for patients and nephrologists. ${ }^{31}$ The VA risk score can be used to identify older adults at low risk of development of ESRD in the next year. In consultation with the individual, clinicians may elect to defer ESRD preparation and access placement for individuals in the lower and middle tertiles (risk score $<2.9 \%$ ). Individuals with low likelihood of ESRD can avoid the potential risks of vascular access placement (e.g., vascular insufficiency, steal syndrome, and high-output heart failure). This is especially important given recent evidence from the Initiating Dialysis Early and Late Study indicating no benefit of "early" initiation. ${ }^{32}$ Alternatively, individuals at higher risk of ESRD (top quintile, risk score $>5.6 \%$, observed risk 14\%) may consider preparation for ESRD and placement of dialysis access. This study does not aim to define when dialysis should be initiated but to provide a tool to better inform the planning for dialysis, but it is only one tool; clinical decisions at the individual level need to take into account 
the circumstances for each individual. Finally, research is necessary to assess the ability of these models to improve long-term outcomes in advanced CKD and increase rates of appropriate vascular access placement.

\section{CONCLUSION}

In summary, a model was developed and validated to predict risk for ESRD at 1 year in elderly adults with advanced CKD. The score uses commonly available clinical variables and may be a tool for risk stratification that individuals and physicians can use to inform decisions regarding planning for ESRD in older adults with advanced CKD.

\section{ACKNOWLEDGMENTS}

The paper was presented at the 2011 American Society of Nephrology annual meeting, Philadelphia, Pennsylvania. Dr. Drawz had full access to all the data in the study and takes responsibility for the integrity of the data and the accuracy of the data analysis.

Conflict of Interest: The study was supported in part through a Career Development Award K23DK087919 (PED) from the National Institute of Diabetes and Digestive and Kidney Diseases (NIDDK). The content is solely the responsibility of the authors and does not necessarily represent the official views of the NIDDK or the National Institutes of Health (NIH). Drs. Drawz and Rahman report receiving grant support from NIH.

All other authors report no conflicts of interest.

Author Contributions: Study concept and design: Drawz, Goswami, Azem, Babineau, Rahman. Acquisition of data: Drawz, Goswami, Azem, Rahman. Analysis and interpretation of data: Drawz, Goswami, Azem, Babineau, Rahman. Preparation of Manuscript: Drawz. Final editing of manuscript: Drawz, Goswami, Azem, Babineau, Rahman.

Sponsor's Role: None.

\section{REFERENCES}

1. Coresh J, Selvin E, Stevens LA et al. Prevalence of chronic kidney disease in the United States. JAMA 2007;298:2038-2047.

2. Drawz P, Rahman M. In the clinic. Chronic kidney disease. Ann Intern Med 2009;15:ITC2-1-15; quiz ITC2-16.

3. Drawz PE, Babineau DC, Rahman M. Metabolic complications in elderly adults with chronic kidney disease. J Am Geriatr Soc 2012;60:310-315.

4. O'Hare AM, Choi AI, Bertenthal D et al. Age affects outcomes in chronic kidney disease. J Am Soc Nephrol 2007;18:2758-2765.

5. Levin A, Djurdjev O, Beaulieu M et al. Variability and risk factors for kidney disease progression and death following attainment of stage 4 CKD in a referred cohort. Am J Kidney Dis 2008;52:661-671.

6. O'Hare AM, Batten A, Burrows NR et al. Trajectories of kidney function decline in the 2 years before initiation of long-term dialysis. Am J Kidney Dis 2012;59:513-522.

7. Clinical practice guidelines for vascular access. Am J Kidney Dis 2006;48 (Suppl 1):S176-S247.

8. Levey AS, Greene T, Kusek J et al. A simplified equation to predict glomerular filtration rate from serum creatinine. J Am Soc Nephrol 2000;11:A0828.

9. Borzecki AM, Wong AT, Hickey EC et al. Identifying hypertension-related comorbidities from administrative data: What's the optimal approach? Am J Med Qual 2004;19:201-206.

10. Szeto HC, Coleman RK, Gholami P et al. Accuracy of computerized outpatient diagnoses in a Veterans Affairs general medicine clinic. Am J Manag Care 2002;8:37-43.
11. Tangri N, Stevens LA, Griffith J et al. A predictive model for progression of chronic kidney disease to kidney failure. JAMA 2011;305: 1553-1559.

12. Pencina MJ, D'Agostino RB Sr, D'Agostino RB Jr et al. Evaluating the added predictive ability of a new marker: From area under the ROC curve to reclassification and beyond. Stat Med 2008;27:157-172 discussion 207112.

13. Goeman JJ. L1 penalized estimation in the Cox proportional hazards model. Biom J 2010;52:70-84.

14. Agarwal R, Bunaye Z, Bekele DM et al. Competing risk factor analysis of end-stage renal disease and mortality in chronic kidney disease. Am J Nephrol 2008;28:569-575.

15. Johnson ES, Thorp ML, Platt RW et al. Predicting the risk of dialysis and transplant among patients with CKD: A retrospective cohort study. Am J Kidney Dis 2008;52:653-660.

16. Johnson ES, Thorp ML, Yang X et al. Predicting renal replacement therapy and mortality in CKD. Am J Kidney Dis 2007;50:559-565.

17. Conway B, Webster A, Ramsay G et al. Predicting mortality and uptake of renal replacement therapy in patients with stage 4 chronic kidney disease. Nephrol Dial Transplant 2009;24:1930-1937.

18. Foley RN, Murray AM, Li S et al. Chronic kidney disease and the risk for cardiovascular disease, renal replacement, and death in the United States Medicare population, 1998 to 1999. J Am Soc Nephrol 2005;16:489-495.

19. Hemmelgarn BR, James MT, Manns BJ et al. Rates of treated and untreated kidney failure in older vs younger adults. JAMA 2012;307:2507-2515.

20. Hallan SI, Ritz E, Lydersen S et al. Combining GFR and albuminuria to classify CKD improves prediction of ESRD. J Am Soc Nephrol 2009;20:1069-1077.

21. Keane WF, Zhang Z, Lyle PA et al. Risk scores for predicting outcomes in patients with type 2 diabetes and nephropathy: The RENAAL study. Clin J Am Soc Nephrol 2006;1:761-767.

22. Landray MJ, Emberson JR, Blackwell L et al. Prediction of ESRD and death among people with CKD: The Chronic Renal Impairment in Birmingham (CRIB) prospective cohort study. Am J Kidney Dis 2010;56:1082-1094.

23. Shlipak MG, Katz R, Kestenbaum B et al. Clinical and subclinical cardiovascular disease and kidney function decline in the elderly. Atherosclerosis 2009;204:298-303.

24. Bang H, Vupputuri S, Shoham DA et al. SCreening for Occult REnal Disease (SCORED): A simple prediction model for chronic kidney disease. Arch Intern Med 2007;167:374-381.

25. Kshirsagar AV, Bang H, Bomback AS et al. A simple algorithm to predict incident kidney disease. Arch Intern Med 2008;168:2466-2473.

26. Norris KC, Greene T, Kopple J et al. Baseline predictors of renal disease progression in the African American Study of Hypertension and Kidney Disease. J Am Soc Nephrol 2006;17:2928-2936.

27. Halbesma N, Jansen DF, Heymans MW et al. Development and validation of a general population renal risk score. Clin J Am Soc Nephrol 2011;6:1731-1738.

28. Hippisley-Cox J, Coupland C. Predicting the risk of chronic kidney disease in men and women in England and Wales: Prospective derivation and external validation of the QKidney Scores. BMC Fam Pract 2010;11:49.

29. Conway PH, Clancy C. Comparative-effectiveness research-implications of the Federal Coordinating Council's report. $\mathrm{N}$ Engl J Med 2009;361:328-330.

30. McGinn TG, Guyatt GH, Wyer PC et al. Users' guides to the medical literature: XXII: How to use articles about clinical decision rules. EvidenceBased Medicine Working Group. JAMA 2000;284:79-84.

31. Schell JO, Patel UD, Steinhauser KE et al. Discussions of the kidney disease trajectory by elderly patients and nephrologists: A qualitative study. Am J Kidney Dis 2012;59:495-503.

32. Cooper BA, Branley P, Bulfone L et al. A randomized, controlled trial of early versus late initiation of dialysis. N Engl J Med 2010;363:609-619.

\section{APPENDIX 1. VETERANS AFFAIRS RISK SCORE FORMULA}

To calculate risk of end-stage renal disease at 1 year, calculate L as follows:

$\mathrm{L}=2.373310+0.2334318 \times$ index estimated glomerular filtration rate $(\mathrm{eGFR})-2.108974 \times 1$ if positive 
for congestive heart failure $(\mathrm{CHF})+0.09131896$ $\times$ age $-0.7468557 \times$ albumin $-0.4961119 \times$ potassium + $0.01726844 \times$ average last 5 systolic blood pressure measurements $+0.1402834 \times$ index eGFR $\times 1$ if positive for CHF-0.006568763 $\times$ index eGFR $\times$ Age

Risk of ESRD at 1 year is then calculated a: $1-0.983^{\wedge}$ $\left(2.71828^{\wedge} \mathrm{L}\right)$

\section{SUPPORTING INFORMATION}

Additional Supporting Information may be found in the online version of this article:
Table S1. Hazard ratios for final model from Cox proportional hazards regression and competing risks regression.

Please note: Wiley-Blackwell is not responsible for the content, accuracy, errors, or functionality of any supporting materials supplied by the authors. Any queries (other than missing material) should be directed to the corresponding author for the article. 